\title{
228. Tumoren nach Nierentransplantation
}

\author{
F. Harder, J. Landmann und G. Thiel \\ Departement für Chirurgie, Kantonsspital, $\mathrm{CH}-4031$ Basel
}

\section{Malignant Tumors After Kidney Transplantation}

Summary. Of 271 patients with 304 kidney transplants, between 1967 and 1981, $20(7 \%)$ have developed a malignant tumor and 7 patients $(2.6 \%)$ have died of it. A minimal time interval for tumor induction has to be assumed. Of 154 patients under immunosuppression with a functioning graft for over 1 year, 19 have developed a tumor. The figure of $12.3 \%$ is probably more realistic than the usual $5 \%-7 \%$ incidence, based on all transplanted patients. Of these 154 patients, $6(3.9 \%$ died of their tumor that is $15.4 \%$ of all deaths among the 154 patients exposed to immunosuppression for over 1 year.

Key words: Malignant tumor - Kidney transplantation - Immunosuppression - Incidence.

Zusammenfassumg. 20 von 271 Patienten mit 304 Nierentransplantationen zwischen 1967 und 1981 haben ein Malignom entwickelt. Von diesen $7 \%$ der 271 Patienten sind 7 oder 2,6\% am Tumor gestorben. Da eine minimale Induktionszeit zur Tumorauslösung anzunehmen ist, wurden nur jene Patienten berücksichtigt, die mindestens $1 \mathrm{Jahr}$ mit dem Transplantat unter Immunsuppression standen : 19 Tumoren bei 154 Patienten oder $12,3 \%$, eine wohl realistischere Zahl als die üblichen $5-7 \%$, die sich auf alle Transplantierten überhaupt beziehen. 6 der 154 erliegen ihrem Tumor $(3,9 \%$ statt $2,6 \%$ oder $15,4 \%$ aller 39 Todesfälle bei diesen 154 Patienten. Die Tumoren waren: 9 viscerale, 7 Hauttumoren, 4 Lymphome.

Schliisselwörter: Malignom - Nierentransplantation - Immunsuppression - Incidenz.

\section{Die akute Pankreatitis als Komplikation nach Nierentransplantation}

\author{
P. Heesen, H. D. Jakubowski, H. Goebell, W. Niebel und F. W. Eigler
}

Abteilung für Allgemeine Chirurgie, Chirurgische Universitätsklinik, Abteilung für Gastroenterologie, Medizinische Universitätsklinik, Klinikum der GHS Essen, Hufelandstraße 55, D-3000 Essen.

\section{Acute Pancreatitis After Kidney Transplantation}

Summary. Among the 501 kidney recipients at our centre from 1971 to 1983 we found 8 patients with posttransplantation pancreatitis $(1.4 \%)$. The aetiology of pancreatitis after transplantation is still not clear, although immunosuppressive therapy, especially steroids, seem to be an important factor in inducing pancreatitits. The treatment of pancreatitis in those patients whose transplanted kidneys function well is therefore more difficult. Our experience shows that steroid therapy can continue when there are strong indications for it, but at reduced dosages.

Key words: Transplantation - Pancreatitis - Steroids - Therapy.

Zusammenfassung. Bei 501 allogenen Leichennierentransplantationen von 1971 bis 1983 trat 8mal postoperativ eine akute Pankreatitis auf $(=1,4 \%)$. Die Letalität war mit 5 von 8 Patienten hoch. Ätiologisch wird die immunsuppressive Therapie, insbesondere hohe Steroidgaben, verantwortlich gemacht. Wir sahen im eigenen Krankengut bei hoher Corticosteroidgabe gleichhäufiges Auftreten wie bei niedriger Dosage. Diagnost isch war bei 3 von 8 Patienten die Sonografie entscheidend. Therapeutisch erscheint uns ein vollständiger Abbruch der immunsuppressiven Therapie bei ausreichender Transplantatfunktion nicht gerechtfertigt. Die Indikation zur Weitergabe der glucocorticoidhaltigen Medikamente muß jedoch streng gestellt werden.

Schliusselwörter: Nierentransplantation - Pankreatitis - Corticoide - Therapie. 